\title{
Obstructive Sleep Apnea: A Biomedical Perspective
}

\author{
Ramasamy Chidambaram* \\ Department of Prosthodontics, Faculty of Dentistry, 2d Floor, SEGI University, No.9, Jalan Teknologi, Taman Sains Selangor, Kota Damansara, PJU 5, 47810 Petaling Jaya, \\ Selangor, MALAYSIA. \\ Phone no: +600164724370 \\ E-mail: dr.ramasamyc@gmail.com \\ DOI: 10.5530/jyp.2019.11.68
}

Sleep is essential for the maintenance of physical and psychological health. Sleep-related breathing disorders or sleep-disordered breathing are characterized by abnormal respiration during sleep. "Sleep-related disorders have been classified into the following groups: Obstructive Sleep Apnea (OSA), central sleep apnea, sleep-related hypoventilation and sleep-related hypoxemia." Obstructive Sleep Apnea (OSA) is a sleep disorder characterized by multiple interruptions in breathing during sleep caused by temporary obstructions in the airway. Although often neglected, OSA is a common sleep-related breathing disorder in adults as well as in children. It is unfortunate that currently, one billion people worldwide suffer from OSA and the number is expected to increase in the near future. The number of individuals affected by OSA has been reported to be the highest in China, followed by the USA, Brazil and India. ${ }^{2}$ This high prevalence of OSA represents a heavy burden on public health and warrants the development of novel approaches for the diagnosis and treatment of OSA.

Family Physicians (FPs) are, from the diagnostic perspective, the frontliners and serve as key players during the process of diagnosis in case of OSA. The typical symptoms of OSA include loud snoring, apneic episodes and daytime sleepiness. Despite these prominent symptoms, OSA is unfortunately often anecdotally diagnosed by the partner of the individual suffering from the disorder. ${ }^{3}$ The intention here is not to blame the FPs or the patients, rather to inform that there is a high probability of OSA to go unnoticed in the general population. Honestly, it is quite difficult for the FPs to suspect OSA unless the partner provides them with relevant information in this regard. Therefore, OSA might remain undiagnosed, which could lead to serious consequences, such as diabetes, cardiovascular risks, road accidents and even death in certain cases. Numerous published research works are available not limiting to pioneer guidelines of American Academy of Sleep Medicine (AASM), roles of FPs, sleep specialists and oral physicians. ${ }^{3-5}$ Despite the increase in awareness regarding the disorder, OSA remains to be an under-acknowledged disorder among the general population. For instance, recent American statistics revealed that $80 \%$ of the cases of OSA in the general population go undetected. ${ }^{6}$ In the context of pediatric population, 90 percent remain undiagnosed. ${ }^{7}$ Some important links appear to be missing here. Medical literature has much to comment on OSA, while scarce literature is available in the context of biomedical investigation. In such circumstances, it would be interesting to investigate whether there is any role for a Community Pharmacist (CP) in this regard.

Traditionally, pharmacists have been known just for dispensing drugs. Pharmacists, for far too long, have confined their visions within the walls of pharmacy or hospitals. However, the role of a pharmacist has transformed dramatically over the past three decades. The clinical pharmacy has developed with the concept of pharmaceutical care, which may be defined as a responsible provision of drug therapy for the purpose of achieving definite outcomes to improve the quality of life of the patients.
Pharmaceutical care is, therefore, practiced in collaboration with patients, FPs, nurses and other healthcare providers. The ultimate objective of biomedical care is to enhance the quality of life of patients. Therefore, innovative thinking is required and the young pharmacists and students who wish to create a difference in their communities must undertake the initiative and explore the opportunities available in this field.

Detection of sleep disorders is important in order to facilitate early diagnosis and delivery of treatment, which would, in turn, reduce the impact of the health issues and societal problems associated with such disorders. In comparison to several other primary healthcare settings, community pharmacy is a feasible setting for the screening and intervention services, because of higher public exposure and accessibility. Polysomnography (PSG) is the gold standard in diagnosing OSA. ${ }^{5}$ However, the usage of PSG among CPs is restricted by certain factors, such as intensive labor, cost and educational backgrounds. Ideally, CPs are well positioned to screen the patients who may be at risk for OSA with clinically validated tools. Without appropriate tools, education, or standard measures, it is difficult to monitor sleep disorders. "Currently, CPs are dependent on the self-reporting from patients or on patients' sleep diaries (if indicated), for the management of sleep-related disorders. Most of the previous studies concerning sleep disorders and community pharmacies focused on the screening tools, with only a limited number of studies incorporating patient follow-up and monitoring by CPs" ${ }^{8-11}$ The literature provides surplus data on the validated questionnaires, not limited to Berlin questionnaire (BQ), STOP-Bang Questionnaire (SBQ), STOP Questionnaire (SQ) and Epworth Sleepiness Scale. ${ }^{12}$ A recent exhaustive review concerning validity and sensitivity suggested that SQ and SBQ were reliable tools for the screening of OSA in clinical patients. ${ }^{12}$ The features of a screening questionnaire that render it appropriate vary with the population that is being surveyed. Therefore, it may not be necessary that the questionnaires other than SQ and SBQ, such as BQ and ESS, exhibit less specificity. An Australian study demonstrated that Community Pharmacy Sleep Assessment Tool (CPSAT) may serve as a practical tool for the identification of patients at the risk of developing sleep disorders within the community. ${ }^{13}$ The aforementioned study was the pioneer among the pharmacy-administered studies to develop a standardized questionnaire or scale for the assessment of sleep disorder risk in the community. This provided an opportunity, from the CP's perspective, to diagnose OSA and refer the suspected OSA candidates to FPs for the confirmation of the diagnosis. However, when the selfreporting is poor, there is a higher probability of even the most severe cases of OSA going undiagnosed. The topic, therefore, remains controversial and it is necessary to conduct further studies on validation that are designed specifically for the general population.

On the other side of the situation, there has been inadequate evidence of the effectiveness of models in detecting sleep disorders in specific primary health-care settings. The limited evidence has demonstrated the 
feasibility and effectiveness of CP services. ${ }^{8,9,11,14-16}$ It is possible that through the establishment of a screening program for a targeted population, CPs may be able to improve the identification of high risk of OSA among individuals. Interestingly, evidence from the experience of Australians has suggested that pharmacists have been pro-active in extending their roles for providing clinical services associated with OSA. ${ }^{17}$ Although the Australian pharmacists have demonstrated their efforts in this regard, the rest of the international pharmacy is yet to consider this approach.

Novel interventions are required for providing further accessible primary healthcare options, in order to assist those with/or at the risk of developing sleep disorders, as alternatives to treatment by sleep specialists, who often have extended waiting times for issuing appointments and are usually expensive if additional procedures are required during the treatment. One such procedure is actigraphy. "Actigraphic devices may be worn on the wrist or ankle, relatively unobtrusively, over a longer period of time ranging from days to weeks. In the last 15 years, actigraphy has been viewed as a useful clinical tool, particularly in the context of the evaluation of patients with suspected or confirmed sleep disorders. ${ }^{18}$ The device has been accepted well and is feasible as a home-based monitoring tool for the identification of undiagnosed poor sleep in healthy, self-rated good sleepers. ${ }^{19}$ Recent research has suggested that actigraph may be able to provide objective sleep/wake data to assist the CPs during consultation, which is useful for CPs as it would result in the generation of graphical data and most importantly, it would overcome the obstacle of poor self-reporting. ${ }^{20}$ It is important to recognize that actigraphy does not represent a substitute for the in-laboratory PSG in case of indication for in-laboratory testing." Although AASM has provided recommendations regarding the usefulness and classification of the diagnostic methods that serve as alternatives to a complete PSG, no guidelines have been provided regarding the 'screening' and currently, no screening tools have been recommended. ${ }^{4}$ Another issue that may arise is that the pharmacist's referral to FP after a screening process may or may not be followed by the screened patients. As discussed earlier, there are major issues associated with the diagnosis and detection of sleep disorders in the community. In consideration of the low levels of awareness among the public and healthcare professionals, extensive training and implementation of the validated questionnaires in community pharmacies as part of a national or community management strategy could prove to be beneficial. In the best interests of the patients, especially the ones at a high risk of OSA, screening programs in community pharmacies should be undertaken, targeting a large section of the population. It is imperative that a feasibility study is conducted in this regard.

In conclusion, sleep disorders represent a global concern and an emerging public health issue requiring a range of strategies, from public health education to clinical services for the management of these disorders. Since community pharmacy is accessible and provides services to the patients who may not come in contact with the FPs regularly, CPs are often the first care-providers for the individuals suffering from OSA. Including the pharmacist as a member of the sleep-disorder care team allows the opportunity to expand the reach of services spanning screening, referral, monitoring and counseling for the patients with OSA or those at the risk of developing this disorder. Poor sleep may exert a negative impact on energy levels, mood, performance and enjoyment in life. Therefore, it is worth spending at least a few min- utes to reflect and identify the ways in which the quality and quantity of sleep may be improved.

\section{REFERENCES}

1. Burman D. Sleep disorders: Sleep-related breathing disorders. FP Essent. 2017;460:11-21.

2. Benjafield AV, Ayas NT, Eastwood PR, Heinzer R, Ip MSM, Morrell MJ, et al. Estimation of the global prevalence and burden of obstructive sleep apnoea: A literature-based analysis. Lancet Respir Med. 2019;7(8):687-98.

3. Ramasamy C. Good news: Dentists are competent in diagnosing undiagnosed sleep apnea. J Coll Physicians Surg Pak. 2017;27(5):321.

4. Kapur VK, Auckley DH, Chowdhuri S, Kuhlmann DC, Mehra R, Ramar K. Clinical practice guideline for diagnostic testing for adult obstructive sleep apnea: An American Academy of Sleep Medicine clinical practice guideline. J Clin Sleep Med. 2017;13(3):479-504.

5. Ramasamy C. Good news: Family medicine specialists have more oppor tunity to diagnose undiagnosed sleep apnea in children. Malays $\mathrm{J}$ Med Sci. 2018;25(5):160-61.

6. American sleep apnea association. Sleep apnea information for clinicians, 2019. Available from: https://www.sleepapnea.org/learn/sleep-apnea-informationclinicians/

7. Alexander N, Boota A, Hooks K, White JR. Rapid maxillary expansion and adenotosillectomy in 9 year old twins with pediatric obstructive sleep apnea syndrome: an interdisciplinary effort. J Am Osteopath Assoc.2019; 119(2):126-34.

8. Tran A, Fuller JM, Wong KK, Krass I, Grunstein R, Saini B: The development of a sleep disorder screening program in Australia community pharmacy. Pharm World Sci. 2009;31(4):473-80.

9. Hersberger KE, Renggli VP, Nirkko AC, Mathis J, Schwegler K, Bloch KE: Screening for sleep disorders in community pharmacies-evaluation of a campaign in Switzerland. J Clinical Pharmacy and Therapeutics. 2006;31(1):35-41.

10. Schwegler K, Klaghofer R, Nirkko AC, Mathis J, Hersberger KE, Bloch KE: Sleep and wakefulness disturbances in Swiss pharmacy customers. Swiss Med Weekly 2006;136(9-10):149-54.

11. Fuller JM, Wong KK, Krass I, Grunstein R, Saini B: Sleep disorders screening, sleep health awareness and patient follow-up by community pharmacists in Australia. Patient Educ Couns. 2011;83(3):325-35.

12. Amra B, Rahmati B, Soltaninejad F, Feizi A. Screening questionnaires for obstructive sleep apnea an updated systematic review. Oman Med J. 2018;33(3):18492.

13. Kashyap K, Nissen L, Smith S, Douglas J, Kyle G. Can a community pharmacy sleep assessment tool aid the identification of patients at risk of sleep disorders in the community: A pilot study. Integr Pharm Res Pract. 2012;2012(1):3-12.

14. Perauddin C, Vaillant ML, Pelletier-Fleury N. Cost-Effectiveness of a Community Pharmacist-Led Sleep Apnea Screening Program - A Markov Model. Plos One 2013;8(6):e63894.

15. Noor ZM, Smith AJ, Smith SS, Nissen LM. A study protocol: A community pharmacy-based intervention for improving the management of sleep disorders in the community settings. BMC Health Serv Res. 2014;14(1):74.

16. Perraudin C, Fleury B, Pelletier-Fleury N. Effectiveness of intervention led by a community pharmacist for improving recognition of sleep apnea in primary care--a cohort study. J Sleep Res. 2015;24(2):167-73.

17. Hanes CA, Wong KK, Saini B. Clinical services for obstructive sleep apnea patients in pharmacies: The Australian experience. Int J Clin Pharm. 2014;36(2):460-8.

18. Smith MT, McCrae CS, Cheung J, Martin JL, Harrod CG, Heald JL, et al. Use of actigraphy for the evaluation of sleep disorders and circadian rhythm sleepwake disorders: An American academy of sleep medicine clinical practice guideline. J Clin Sleep Med. 2018;14(7):1231-7.

19. Noor ZM, Smith AJ, Smith SS, Nissen LM. Feasibility and acceptability of wrist actigraph in assessing sleep quality and sleep quantity: A home-based pilot study in healthy volunteers. Health. 2013;5(8A2):63-72.

20. Noor ZM, Smith AJ, Smith SS, Nissen LM. A feasibility study: Use of actigraph to monitor and follow-up sleep/wake patterns in individuals attending community pharmacy with sleeping disorders. J Pharm Bioallied Sci. 2016;8(3):173-80. 